\title{
Ischemic Conditioning Ameliorated Hypertension and Vascular Remodeling of Spontaneously Hypertensive Rat via Inflammatory Regulation
}

\author{
Yu Gao ${ }^{1,2}$, Changhong Ren ${ }^{2,3}$, Xiaohua $\mathrm{Li}^{2,3}$, Wantong Yu', Sijie $\mathrm{Li}^{2,3}$, Haiyan $\mathrm{Li}^{2}$, Yan Wang', \\ Dong $\mathrm{Li}^{4}$, Ming Ren ${ }^{1}$, Xunming $\mathrm{Ji}^{1,5^{*}}$
}

${ }^{1}$ Department of Neurology, Xuanwu Hospital, Capital Medical University, Beijing, 10053, China. ${ }^{2}$ Beijing Key Laboratory of Hypoxia Conditioning Translational Medicine, Xuanwu Hospital, Capital Medical, Beijing, 10053, China. ${ }^{3}$ Beijing municipal geriatric medical research center, Beijing, 10053, China. ${ }^{4}$ Peking University Care Health Management Center, Beijing, 100080, China. ${ }^{5}$ Department of Neurosurgery Xuanwu Hospital, Capital Medical, Beijing 100053, China.

[Received February 19, 2020; Revised March 20, 2020; Accepted March 27, 2020]

\begin{abstract}
Vascular remodeling is an initial step in the development of hypertension. Limb remote ischemic conditioning (LRIC) is a physiological treatment that induces endogenous protective effect during acute ischemic injury. However, the impact of long-term LRIC on hypertension, a chronic disease, is unknown. In this study, we aimed to investigate the LRIC effect on blood pressure and vascular remodeling in spontaneously hypertensive rat (SHR) model and patients with prehypertension and early-stage hypertension. LRIC of rats was performed once a day for 6-weeks. Blood pressure, vascular remodeling (cross-sectional area, extracellular deposition, and smooth muscle cell area), inflammation (inflammatory factors, and inflammatory cells) were compared among normotensive Wistar-Kyoto rats (WKY), WKY RIC group, SHR control group, and SHR RIC. Long-term LRCI treatment (twice a day for 4-weeks) was performed on patients with prehypertension or early-stage hypertension. Blood pressure and pulse wave velocity (PWV) were analyzed before and after LRIC treatment. LRIC treatment decreased blood pressure in SHR $(n=9-10)$. LRIC ameliorated vascular remodeling by decreasing cross-sectional area, suppressing deposition of the extracellular matrix, and hypertrophy of smooth muscle cell in conduit artery and small resistance artery $(n=7)$. LRIC decreased proinflammatory factors while increasing the antiinflammatory factors in the circulation $(n=5)$. LRIC decreased circulating monocyte and natural killer T-cell levels $(n=5)$. Furthermore, LRIC treatment decreased blood pressure and improved vascular stiffness in patients $(n=$ 20). In conclusion, long term LRIC could decrease blood pressure and ameliorate vascular remodeling via inflammation regulation. LRIC could be a preventive treatment for people with blood pressure elevation or prehypertension.
\end{abstract}

Key words: hypertension, vascular remodeling, inflammation, limb remote ischemic preconditioning

Hypertension is a leading risk factor for cardiovascular, cerebrovascular, and many other diseases [1], especially cardiovascular and cerebrovascular diseases, which are leading causes of global deaths, according to the World
Health Organization [2]. Vascular remodeling and dysfunction are the main pathological processes preceding the occurrence of hypertension and its complications [3-5]. Vascular remodeling results from

*Correspondence should be addressed to: Dr. Xunming Ji, Department of Neurosurgery, Xuanwu Hospital, Capital Medical University, No. 45, Changchun Street, Xicheng District, Beijing, China. Email: jixm@ccmu.edu.cn.

Copyright: ( 92020 Gao Y et al. This is an open-access article distributed under the terms of the Creative Commons Attribution License, which permits unrestricted use, distribution, and reproduction in any medium, provided the original author and source are credited. 
blood pressure elevation, and progressively becomes a crucial cause of hypertension [6]. However, during vascular remodeling no symptoms except occasional blood pressure elevation are observed. Neither attention nor treatment would be considered until the diagnosis of hypertension is established [7]. Thus, timely therapy is urgent for the prevention and treatment of early-stage vascular remodeling.

Vascular remodeling results initially from passive physiological adaptation to blood pressure changes, then progresses into an active pathological process caused by elevated blood pressure, aging, and several other factors $[8,9]$. This pathological process involves a series of changes, including cell proliferation, migration, transformation, production of the extracellular matrix, and inflammation [5]. An earlier study showed the critical role of inflammation in the pathological changes involved in vascular remodeling [10]. Emerging evidence indicates that infiltrating proinflammatory cells are essential for the migration and infiltration of inflammatory factors [11]. Several inflammatory factors were shown to affect blood pressure and vascular function leading to vascular remodeling and dysfunction [12]. Thus, it is reasonable to hypothesize that by regulating inflammatory cells and their environment might aid in the treatment and prevention of vascular remodeling and hypertensionrelated vascular diseases.

Limb remote ischemic conditioning (LRIC) is a physiological treatment that protects against acute ischemic events and traumatic injury [13, 14]. Chronic remote ischemic conditioning simulates regular exercise and exerts its protective effect via humoral and immunological regulation [14, 15]. Some clinical cases reported that LRIC could decrease blood pressure [16]. However, studies on whether LRIC positively affects chronic vascular remodeling and blood pressure are scant. Considering all the available evidence, we hypothesize that LRIC would exert a protective effect on hypertension-related vascular remodeling, thus delaying vascular stiffness and aging caused by structural remodeling.

In this study, we aimed to address whether LRIC exhibits antihypertensive effect and if LRIC prevents or delays vascular remodeling in a chronic hypertensive pathological condition and the possible underlying mechanism of LRIC protective effect.

\section{MATERIALS AND METHODS}

\section{Animals}

Spontaneously hypertensive rats (SHR) were used as a genetic model of hypertension, which develop hypertension at a very young age. Before the established phase, the blood pressure of SHR increases, and vascular remodeling occurs rapidly from age 4-10 weeks [17]. In this study, we chose the 4-week-old SHR, which has similar blood pressure compared to the Wistar-Kyoto rats (WKY), normotensive controls.

The Animal Care and Use Committee of Xuanwu Hospital, Capital Medical University, China approved all animal experiments which were conducted following the National Institutes of Health guidelines. Forty rats used in this study were purchased from the Vital River Laboratories, Beijing, China, and maintained on a 12-h light/dark cycle with unlimited access to food and water. We assigned randomly $20 \mathrm{WKY}$ to WKY control (WKYCON) and WKY + RIC groups, and 20 SHR to SHR control (SHR-CON) and SHR + RIC groups, respectively.

\section{Limb Remote Ischemic Conditioning}

LRIC was conducted as previously described[18]. Rats were anesthetized with an intraperitoneal injection of sodium pentobarbital $(30 \mathrm{mg} / \mathrm{kg})$, and three cycles of LRIC conducted daily by tightening and releasing of a tourniquet around the upper thigh. Each cycle comprised of $10 \mathrm{~min}$ of tightening (ischemia) and $10 \mathrm{~min}$ of release (reperfusion). The treatment was administered every day for 6-weeks (Fig. 1A).

\section{Blood Pressure Measurements}

The blood pressure was measured weekly, 12 hours after LRIC, with a tail-cuff system (SoftronBP-2010A; Softron, Tokyo, Japan) following the manufacturer's guidelines.

\section{Histological analysis}

The thoracic aorta, second-order mesenteric artery, and brain were harvested and fixed in $4 \%$ paraformaldehyde, dehydrated, and embedded in paraffin. Paraffin sections of $5 \mu \mathrm{m}$ thickness were deparaffinized and washed, stained with hematoxylin and eosin (H\&E), Sirius-red, and Verhoeff's elastic stain (VEG) [19]. For H\&E staining, tissues were incubated with hematoxylin solution for $8 \mathrm{~min}$, rinsed in running water, differentiated in $1 \%$ acid alcohol for $30 \mathrm{sec}$, rinsed in water, and counterstained in eosin solution for $1 \mathrm{~min}$. Sirius-red staining was carried out by incubating tissues with Sirius red for $1 \mathrm{~h}$, washed, and nuclei stained with Mayer's solution followed by washing. For VEG staining, tissue was immersed in Verhoeff's solution for $1 \mathrm{~h}$, differentiated with $2 \%$ ferric chloride for $1 \mathrm{~min}$, washed in water, then treated with $5 \%$ sodium thiosulfate for 1 min, washed, and counterstained with Van Gieson's solution for 3-5 $\mathrm{min}$. Then all slices were dehydrated in 
alcohol, cleared in xylene, and mounted using xylene based mounting medium. Images were viewed and captured using a digital slide scanner (3DHISTECH, UK) and Nikon Ti Eclipse Epi-fl Illuminator (Nikon, Japan). The images were quantified by Image $\mathbf{J}$ software. The quantification of hypertrophic remodeling includes the medial area and vessel wall thickness. The collagen and elastic fiber content were calculated by summing up all positive tissue areas from all measurements of the section [19].

A
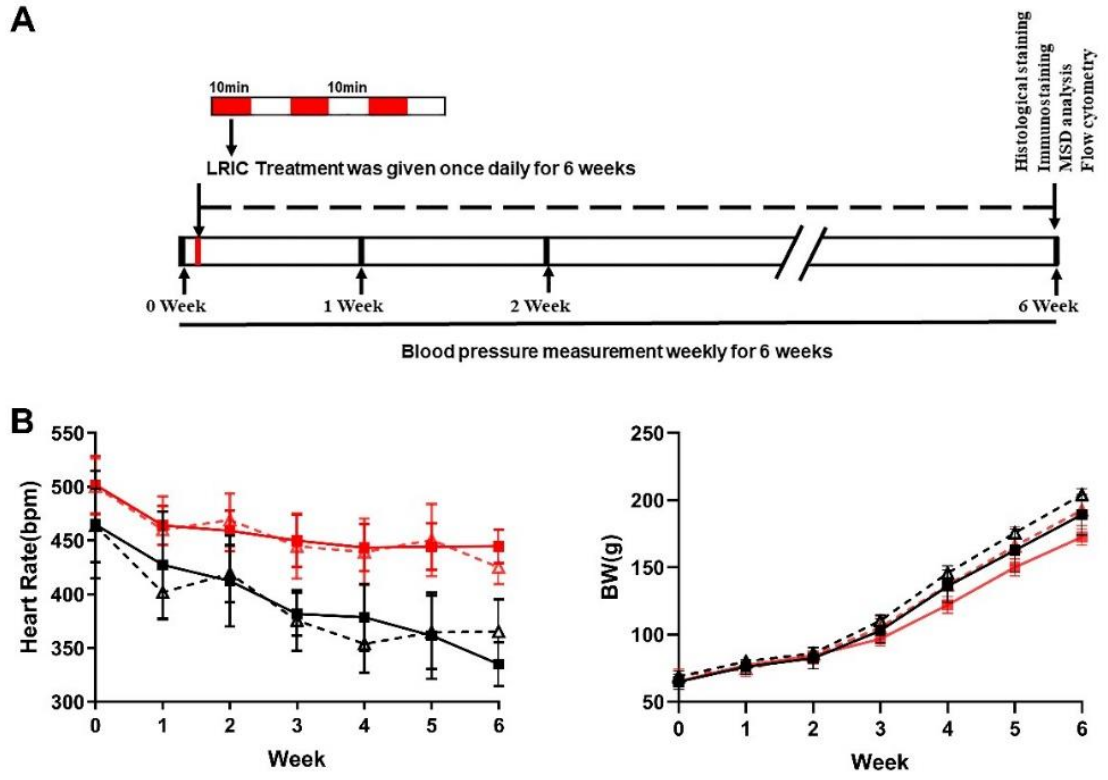

Figure 1. Effect of LRIC on blood pressure in SHR and WKY. (A) Representative sketches of this experiment. (B) Heart rate and the bodyweight of each group. Systolic blood pressure (SBP) (C), diastolic blood pressure (DBP) (D), and mean blood pressure (MBP) (E) of each group over 6-weeks. Data are expressed as mean $\pm \mathrm{SD}$, * $\mathrm{P}<0.05, * * \mathrm{P}<0.01, * * *$ $\mathrm{P}<0.001$, SHR control vs. SHR RIC. \# P $<0.01$, WKY control vs. SHR control, $\mathrm{n}=9$ 10 each group.
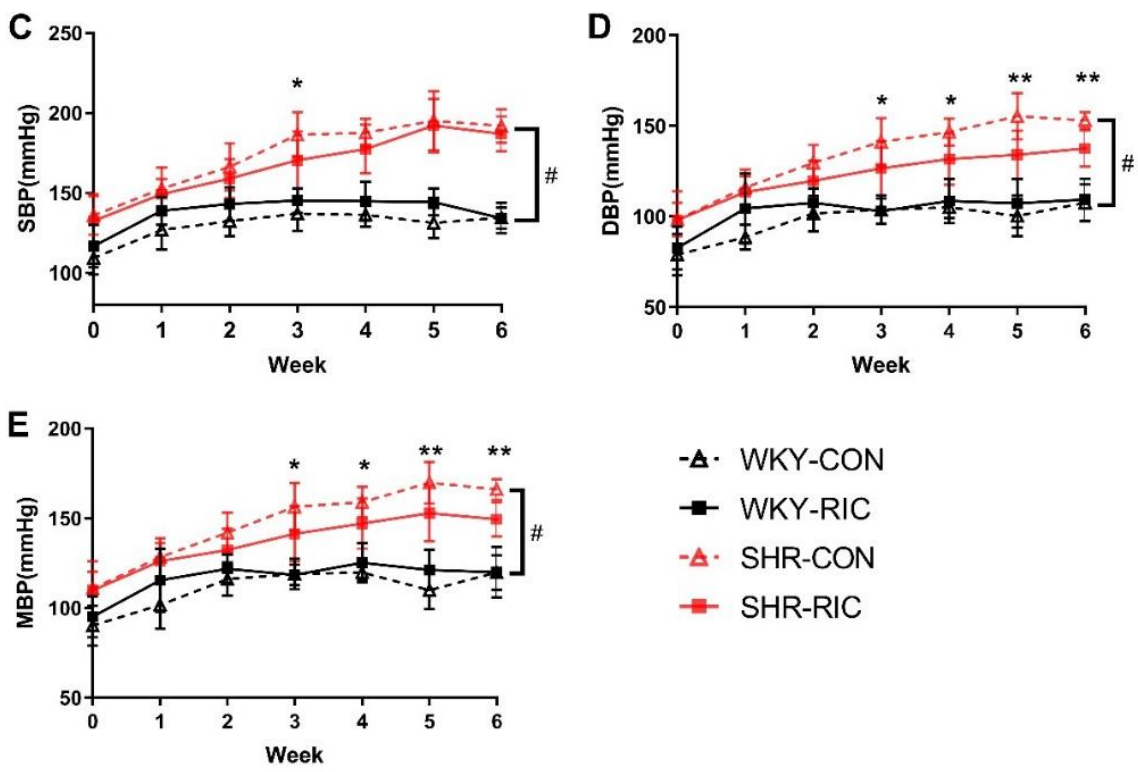

\section{Immunohistochemistry}

Tissue slices were incubated overnight at $4^{\circ} \mathrm{C}$ with antiSMA antibody (1:100, Abcam, US). After washing with PBS, the sections were incubated for $1 \mathrm{~h}$ at $21{ }^{\circ} \mathrm{C}$ with the secondary antibodies. Slides were mounted with an antifade reagent containing DAPI (Molecular Probes,
USA). Fluorescence signals were detected with the Panoramic digital slide scanner (3DHISTECH, UK), and quantified using Image J software. The $\alpha$-SMA expression values were calculated from the total positive area and mean fluorescence intensity. 


\section{Flow Cytometry}

Immunocytes in the blood were analyzed by flow cytometry. Single-cell suspensions were treated with Fc block, washed, and stained with $\mathrm{CD} 3, \mathrm{CD} 4, \mathrm{CD} 8, \mathrm{CD} 45$, CD161a, CD43, CD172a, and their homologous isotypematched negative controls (BD, Franklin Lakes, NJ). Live cells were gated, and a set number of events were acquired using a Fortessa flow cytometer (BD) and analyzed.

\section{Measurement of Inflammatory Factors}

Blood from the inferior vena cava was collected into EDTA coated vacutainer tubes, followed by centrifugation at $1000 \mathrm{~g}$ for $15 \mathrm{~min}$ at $21^{\circ} \mathrm{C}$. Plasma was obtained and stored at $-80^{\circ} \mathrm{C}$ [20]. An ELISA assay for plasma inflammatory factors was conducted with the Meso Scale Discovery (MSD, US) rat inflammation kit following the manufacturer's instructions.

Table 1. Baseline characteristics of recruited patients.

\begin{tabular}{|c|c|}
\hline Characteristics & Value, $n=20$ \\
\hline Age & $57 \pm 8.4$ \\
\hline Male & $9(45)$ \\
\hline Height, Kg & $68.9 \pm 8.8$ \\
\hline Weight, cm & $166.1 \pm 8.3$ \\
\hline BMI $\left(\mathrm{Kg} / \mathrm{m}^{2}\right)$ & $24.8 \pm 2.4$ \\
\hline Systolic blood pressure, $\mathrm{mmHg}$ & $137.2 \pm 9.5$ \\
\hline Diastolic blood pressure, $\mathrm{mmHg}$ & $82.9 \pm 6.9$ \\
\hline Mean blood pressure, $\mathrm{mmHg}$ & $100.8 \pm 6.1$ \\
\hline Heart rate, bpm & $73.5 \pm 8.1$ \\
\hline \multicolumn{2}{|l|}{ Cholesterol } \\
\hline Total, $\mathrm{mmol} / \mathrm{L}$ & $5.3 \pm 0.8$ \\
\hline LDL, $\mathbf{m m o l} / \mathbf{L}$ & $2.9 \pm 0.6$ \\
\hline HDL, $\mathrm{mmol} / \mathrm{L}$ & $1.4 \pm 0.2$ \\
\hline Triglycerides, mmol/L & $1.9 \pm 0.9$ \\
\hline Creatinine, $\mu \mathrm{mol} / \mathrm{L}$ & $5.8 \pm 0.6$ \\
\hline Fasting blood glucose, $\mathrm{mmol} / \mathrm{L}$ & $4.6 \pm 1.4$ \\
\hline White blood cell count $\left(10^{9}\right)$ & $5.5 \pm 1.1$ \\
\hline
\end{tabular}

\section{Patient recruitment and treatment}

The Institutional Ethics Committee of Xuanwu Hospital approved this investigation. In this study, 20 prehypertensive or early-stage hypertensive subjects not receiving any pharmacological anti-hypertensive treatment were included. The study inclusion criteria were as follows: (1) systolic blood pressure (SBP) between $125-160 \mathrm{mmHg}$ or diastolic blood pressure (DBP) between 80-100 mmHg; (2) age $\geq 18$; (3) essential hypertension; (4) patient not receiving any antihypertensive medication. The exclusion criteria were: (1) patients with severe uncontrolled diabetes; (2) contraindication for remote ischemic preconditioning; (3) life expectancy less than one year; (4) patients with atrial fibrillation or other kinds of arrhythmia; (5) unwillingness for follow-up or poor compliance. (Registration No. NCT04254432, https://register.clinicaltrials.gov)

The baseline characteristics of patients are shown in Table 1. LRCI treatment was administered as described previously, and all patients habituated to the treatment over three days [21]. Bilateral upper limb RIC was performed using an electric auto-control device (patent number ZL200820123637.X, China), which consisted of five alternating cycles of inflation and deflation for 5 minutes, twice daily for 4-weeks, and the inflation pressure was $200 \mathrm{mmHg}$.

Baseline blood pressure and pulse wave velocity (PWV) were measured following the previously described guidelines [1]. Patients were prepared accordingly, and the blood pressure measured by electronic blood pressure monitor (Omron, Japan) and PWV measured by the noninvasive Vascular Screening Device (Omron, Japan). After completion of the LRIC treatment, blood pressure and PWV were measured within 3 days.

\section{Statistical analysis}

Data calculated using SPSS version 19.0 (SPSS, Chicago, IL, USA) software were expressed as mean \pm standard deviation (SD). For animal experiments, differences between groups were analyzed using one-way analysis of variance (ANOVA), and posthoc multiple comparisons were performed using Fisher's LSD where appropriate. Two-way repeated-measures ANOVA was used for analysis of a comparison of blood pressure across time points. For the human trials, differences between groups were analyzed using two-sided paired Student's $t$-test. All analyses were performed with significance set at $\mathrm{P}<0.05$.

\section{RESULTS}

\section{LRIC treatment decreased SHR blood pressure with no effect on heart rate and body weight}

Blood pressure in SHR increased rapidly from the age of 4-10 weeks compared to the WKY group. The mean blood pressure of the two groups was $119.8 \pm 9.6$ and $166.0 \pm$ $5.7 \mathrm{mmHg}$, respectively (Fig. 1E). LRIC treatment decreased SHR blood pressure to certain extent without 
affecting body weight and heart rate (Fig. 1B). The mean blood pressure in the SHR-CON and SHR RIC groups was $166.0 \pm 5.7$ and $149.5 \pm 9.6 \mathrm{mmHg}$, respectively, after a 6-week treatment. LRIC resulted in decreased blood pressure of greater than $10 \mathrm{mmHg}$ compared to SHR-
CON group. The effect of LRIC on blood pressure appeared in the third week of treatment and continued after that (Fig. 1E). However, LRIC could not decrease SHR blood pressure to levels comparable to WKY.
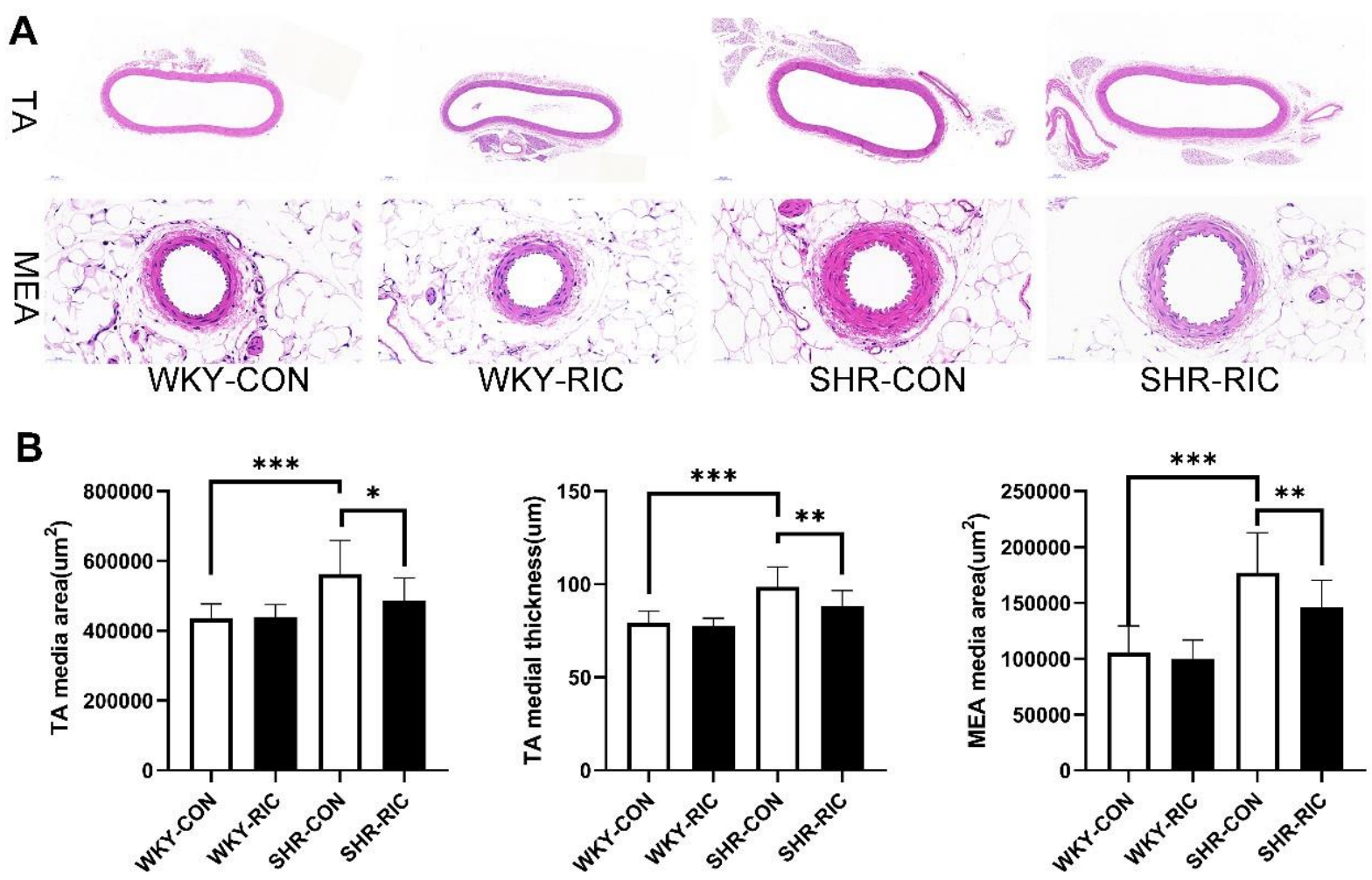

Figure 2. LRIC treatment ameliorated hypertrophic vascular remodeling of conducting artery and small resistance artery. (A) H\&E staining of the thoracic aorta (TA) and second-order mesenteric artery (MEA). (B) Tunica media area and the wall thickness of TA and MEA of each group. Data are expressed as mean $\pm \mathrm{SD}, * \mathrm{P}<0.05, * * \mathrm{P}<0.01, * * * \mathrm{P}<0.001$, WKY-CON vs. SHR-CON, SHR-CON vs. SHR-RIC. $\mathrm{N}=7$ each group.

\section{LRIC treatment ameliorated hypertrophic vascular remodeling of conducting artery and small resistance artery (second-order mesenteric artery)}

Hypertrophy is recognized as the primary cause during the transition from the physiological to pathological vascular remodeling in hypertension [5]. Hypertrophic remodeling is visible primarily in the increased media cross-sectional area and decreased media/lumen ratio. We investigated whether LRIC has a positive effect on vascular remodeling by performing Hematoxylin and Eosin (H\&E) staining on the thoracic aorta and small resistance artery (second-order mesenteric artery). The area of media and wall thickness was evaluated after a 6-week treatment (Fig. 2A). The media area of the thoracic aorta and small resistance artery of SHR increased significantly compared to WKY (Fig. 2B). LRIC treatment decreased media thickness and media area of the thoracic aorta and secondorder mesenteric artery by 10 and $18 \%$, respectively, compared to SHR-CON (Fig. 2B) The LRIC showed a higher beneficial effect on small resistance artery than that on conducting artery (Fig. 2B).

\section{LRIC treatment ameliorated hypertrophic vascular remodeling of brain resistance artery (middle cerebral artery, $M C A$, and basilar artery, $B A)$}

Hypertrophic remodeling causes increased peripheral resistance and decreased blood supply [12]. The resistance of muscular arteries appears to be higher in the cerebral circulation than in other vascular systems [23]. The cerebral resistance artery contributes considerably to 
total cerebral vascular resistance in normal and hypertensive conditions, which further decreases cerebral blood supply and brain dysfunction [22, 23]. We assessed whether LRIC exhibited a similar effect on the cerebral resistance artery compared to systemic by performing $\mathrm{H} \& \mathrm{E}$ staining on the main cerebral resistance artery, middle cerebral artery (MCA), and basilar artery (BA) and compared the media area of each group (Fig. 3A). Cerebral resistance arteries of SHR showed significant hypertrophic remodeling compared to WKY (Fig. 3B). LRIC could ameliorate the hypertrophic vascular remodeling of $\mathrm{MCA}$ and $\mathrm{BA}$, decreased the media area by 17 and $13 \%$, respectively (Fig. 3B). The effect was similar to that on the mesenteric artery.
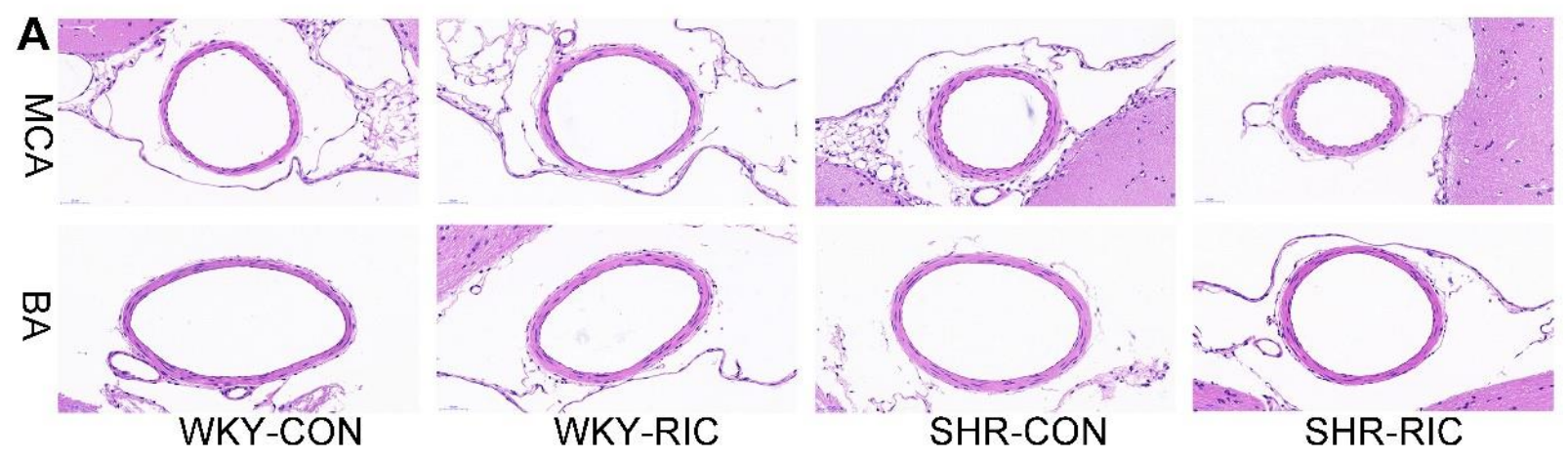

$\mathbf{B}$
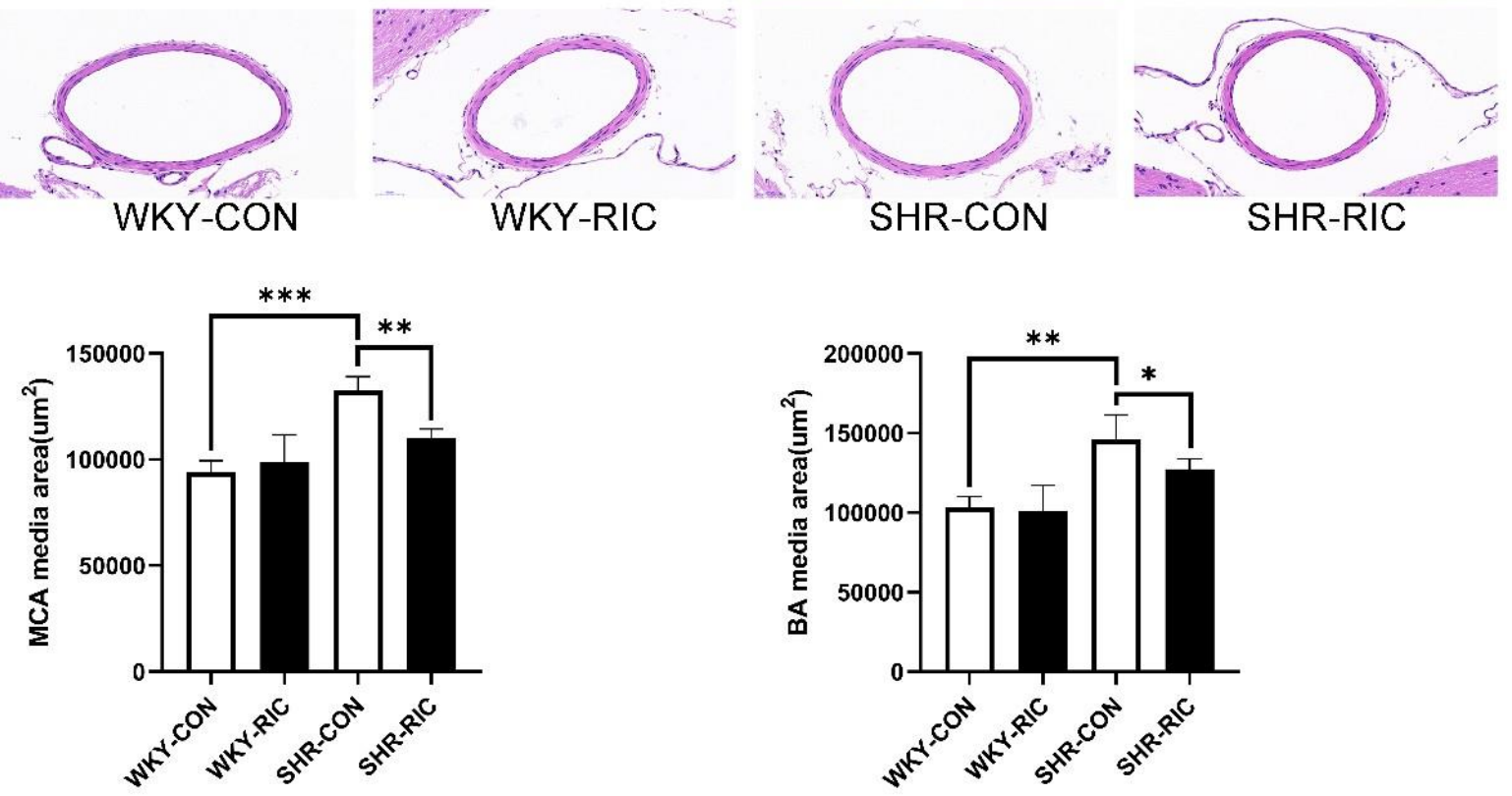

Figure 3. LRIC treatment ameliorated hypertrophic vascular remodeling of middle cerebral artery and basilar artery. (A) H\&E staining of the middle cerebral artery (MCA) and basilar artery (BA). (B) Media area of MCA and BA of each group. Data are expressed as mean $\pm \mathrm{SD}, * \mathrm{P}<0.05, * * \mathrm{P}<0.01, * * * \mathrm{P}<0.001$, WKY-CON vs. SHR-CON, SHR-CON vs. SHR-RIC. $\mathrm{N}=5$ each group.

\section{LRIC suppress extracellular matrix production of the conducting artery and small resistance artery}

Vascular hypertrophy involves extracellular matrix production, smooth muscle cell hyperplasia, and hypertrophy [24]. We investigated their effect on the cross-sectional area by examining levels of different extracellular matrices, collagen I, collagen III, and elastic fiber from each group (Fig. 4A and B; Fig. 5A and B). The results showed markedly increased deposition of collagen I, collagen III, and elastic fiber in the SHR-CON group compared to the WKY-CON (Fig. 4C; Fig. 5C). LRIC decreased both collagen I, and collagen III deposition in TA and MEA (Fig. 4C; Fig. 5C). Collagen levels decreased by 30 and $15 \%$ in MEA and TA, respectively, and LRIC showed a negligible 3\% decrease in the elastic fiber of MEA (Fig. 5C). In contrast, LRIC decreased by nearly $15 \%$ of elastic fiber deposition in TA (Fig. 4C). Altogether, LRIC suppresses the production of collagen in the small resistance artery considerably with no significant effect on the elastic fiber. LRIC decreased $15 \%$ of all extracellular component production in the conduit artery.

\section{LRIC treatment suppressed smooth muscle cell hypertrophy of small resistance artery}

Smooth muscle cell (SMC) hypertrophy and hyperplasia were reported in early-stage hypertension and play a significant role in pathological remodeling [5]. 
Hypertrophy is considered a critical cellular response process in early hypertension [25]. We investigated whether LRIC would affect SMC by immunostaining of $\alpha$-SMA in the vascular smooth muscle cell (Fig. 6A). Results showed that the area and expression of $\alpha$-SMA increased significantly in the SHR-CON compared to WKY-CON (Fig. 6B, C, D, E). The area and fluorescence intensity of SMC decreased by $20 \%$ and $5 \%$ in MEA and TA, respectively. $\alpha$-SMA expression decreased by $17 \%$ and $7 \%$ in MEA and TA, respectively. These results indicated that LRIC could ameliorate SMC hypertrophy in the small resistance artery with no effect on the conducting artery.
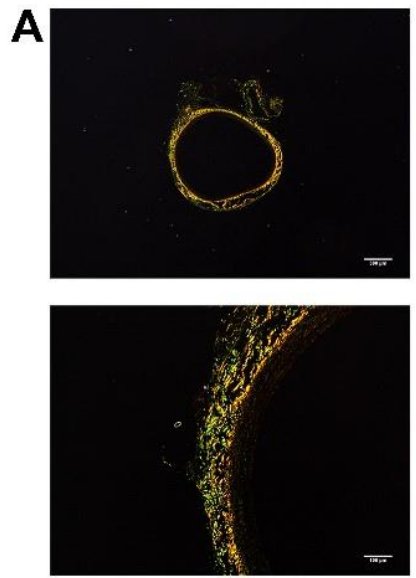

B

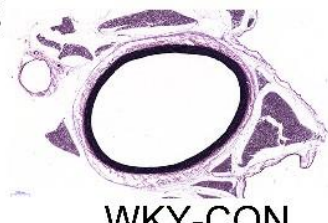

WKY-CON

C

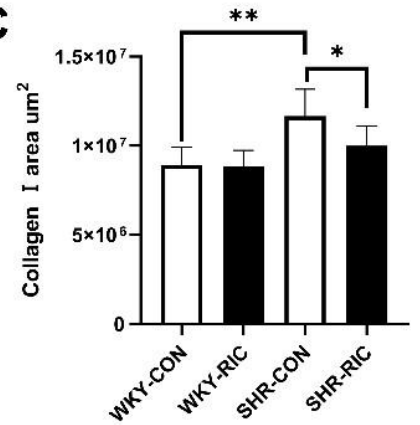

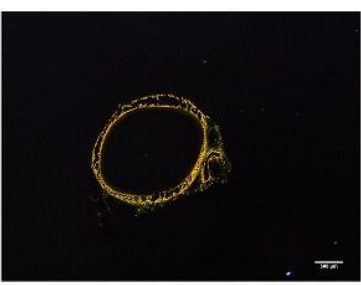
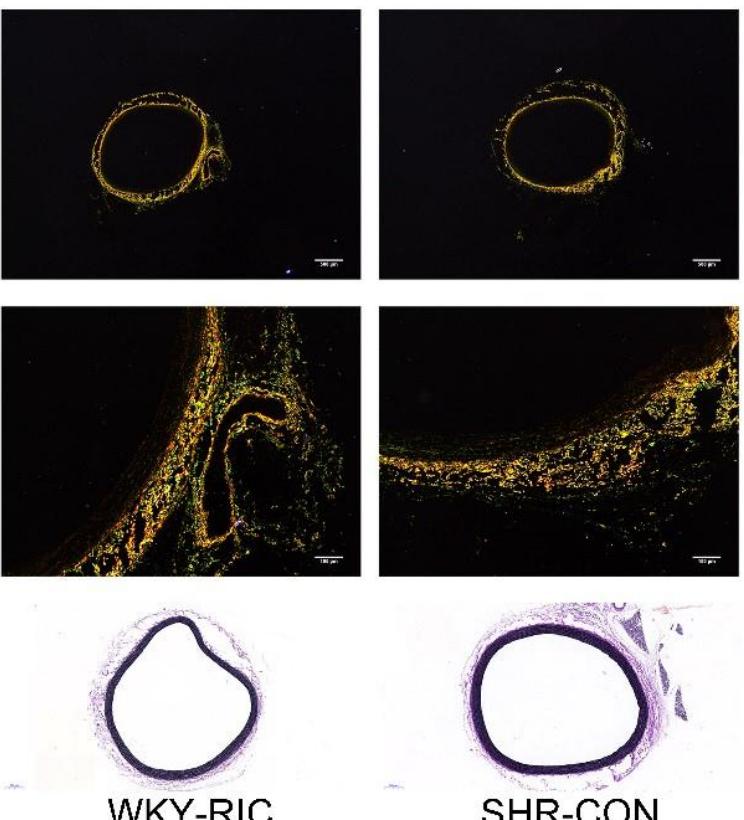

SHR-CON
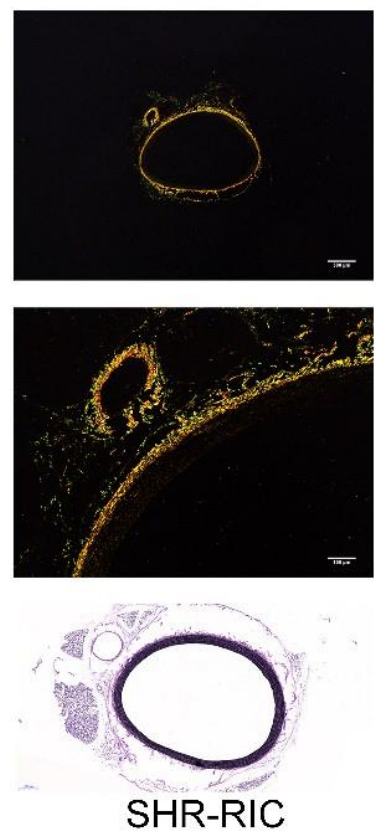

Figure 4. LRIC suppressed the production of the extracellular matrix of the conducting artery. Sirius-red (A) and VEG staining (B) of TA. (C) Collagen I, Collagen III, and elastic fiber area of TA. Data are expressed as mean $\pm \mathrm{SD}, * \mathrm{P}<0.05, * *$ $\mathrm{P}<0.01, * * * \mathrm{P}<0.001, \mathrm{WKY}-\mathrm{CON}$ vs. SHR-CON, SHR-CON vs. SHR-RIC. $\mathrm{N}=7$ each group.

\section{LRIC treatment regulated circulating pro-inflammatory and anti-inflammatory factors}

Dysregulation of circulating inflammatory factors was shown to play a central role in initiating blood pressure elevation and early-stage vascular remodeling [12]. The effect of LRIC on circulating inflammatory factors was investigated by determining the levels of hypertension- related pro- and anti-inflammatory factors using MSD. The results showed significantly increased proinflammatory factors CXCL1, TNF $\alpha$, and IL-1 $\beta$ in SHR (Fig. 7A), compared to WKY, while the antiinflammatory factors IL-10 and IL-13 decreased markedly (Fig. 7B). The levels of other inflammatory cytokines, IFN $\gamma$, IL-5, IL-6, and IL-4, were not significantly different between SHR vs. WKY (Fig. 7A). 
LRIC decreased pro-inflammatory factors CXCL1, $\mathrm{TNF} \alpha$, and IL-1 $\beta$ (Fig. 7A) and increased antiinflammatory factors IL-10 and IL-13 in SHR (Fig. 7B).
LRIC treatment did not affect the levels of IFN $\gamma$, IL-4, IL5, and IL-6 (Fig. 7 A and B).
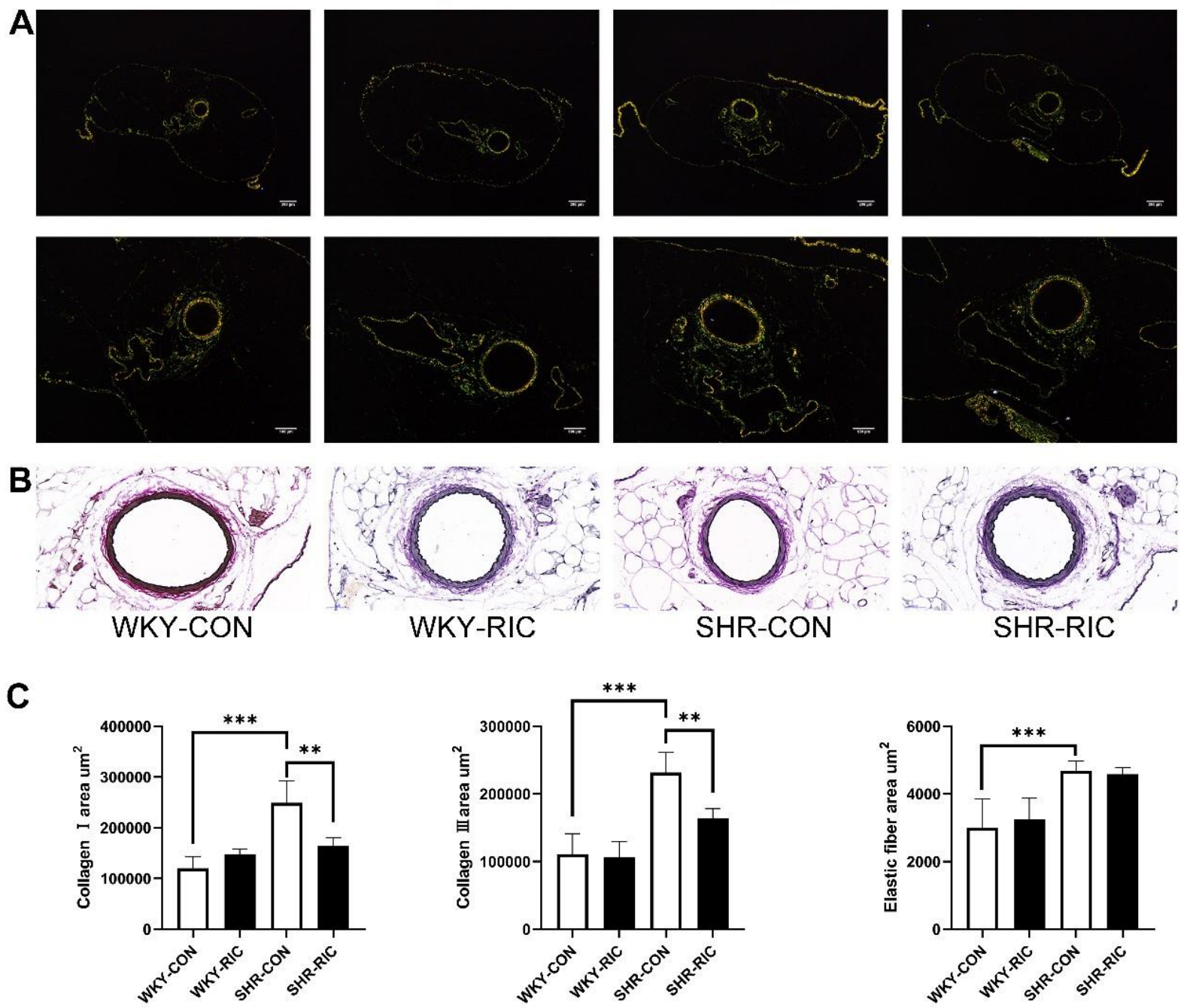

Figure 5. LRIC suppressed the production of the extracellular matrix of the small resistance artery. Sirius-red (A) and VEG staining (B) of MEA. (C) Collagen I, Collagen III, and elastic fiber area of MEA. Data are expressed as mean $\pm \mathrm{SD}, * \mathrm{P}<0.05, * *$ $\mathrm{P}<0.01, * * * \mathrm{P}<0.001$, WKY-CON vs. SHR-CON, SHR-CON vs. SHR-RIC. $\mathrm{N}=7$ each group.

\section{LRIC treatment regulated inflammatory immunocytes in circulation}

Hypertrophic remodeling is initiated by vascular infiltration of inflammatory cells, especially macrophages [11]. Inflammatory factors secreted by immunocytes are critical components involved in recruitment and infiltration during the pathogenesis of vascular remodeling in hypertension [26]. Thus, we analyzed the immunocyte levels in each group to investigate whether LRIC affects immunity (Fig. 8A). We found an increased number of all types of monocytes in SHR compared to
WKY (Fig. 8B). In addition, we identified significantly increased natural killer T cells (NKT) in SHR (Fig. 8 B). LRIC decreased levels of monocytes and natural killer $\mathrm{T}$ cells significantly (Fig. 8B).

\section{Long term LRIC decreased blood pressure and ameliorated conducting artery $P W V$}

We investigated whether LRIC exhibits similar effects in humans by conducting a pilot study on patients with preand early-stage hypertension. Twenty patients completed the 4-week ischemic conditioning treatment. The results 
showed that a 4-week LRIC treatment was well tolerated: 5476 out of 5600 cycles were completed in total. Eleven and nine subjects completed over 90 and $80 \%$ cycles, respectively. The percentage of completed cycles for each week was $98 \%, 97 \%, 96 \%$, and $94 \%$, respectively. The primary reason for uncompleted cycles was intense workouts, while other reasons are, discomfort due to cuff pressure, or lack of adherence to schedule (Fig. 9). The systolic blood pressure (SBP), diastolic blood pressure (DBP), and mean blood pressure (MBP) decreased by $10.2,5.4$, and $6.78 \mathrm{mmHg}$, respectively, after a 4-week LRIC treatment compared to their baseline blood pressure. PWV decreased $101 \mathrm{~cm} / \mathrm{s}$ on average (Fig. 10). The results showed that long-term LRIC could decrease blood pressure and ameliorate the large artery stiffness in patients with pre- or early-stage hypertension.
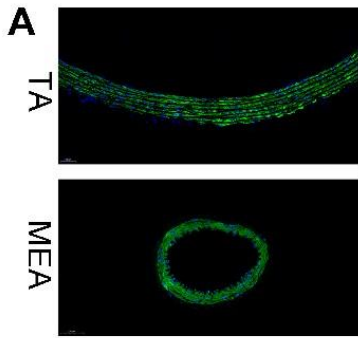

WKY-CON
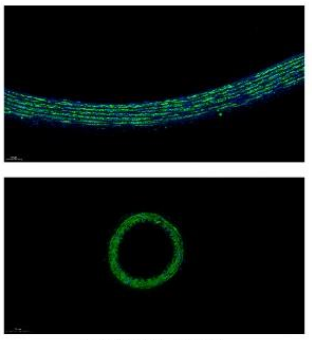

WKY-RIC
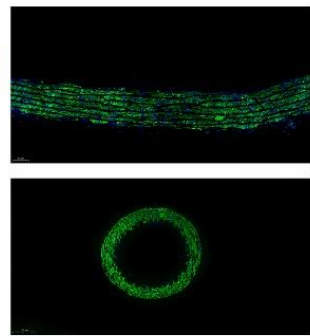

SHR-CON
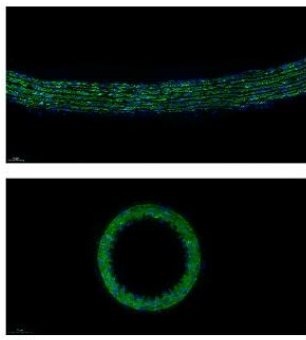

SHR-RIC
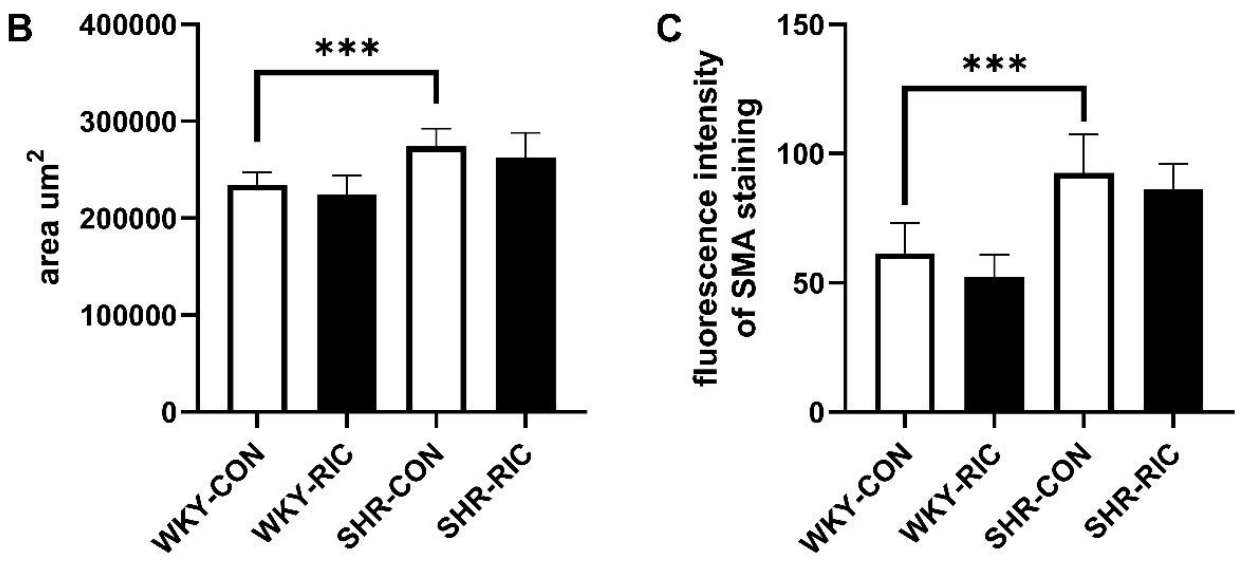

D

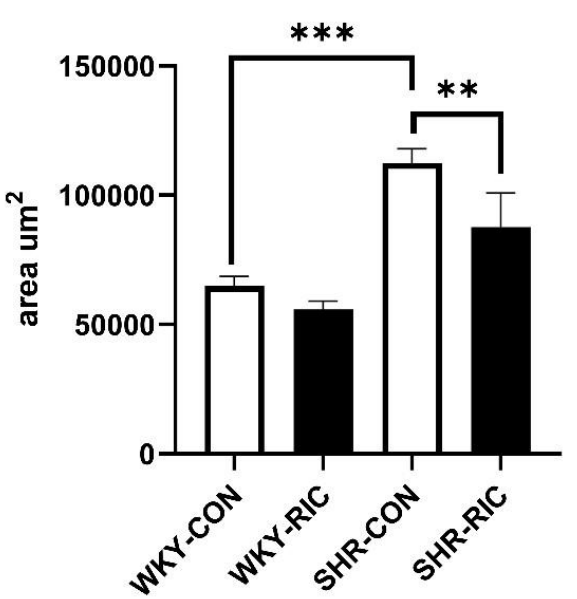

E

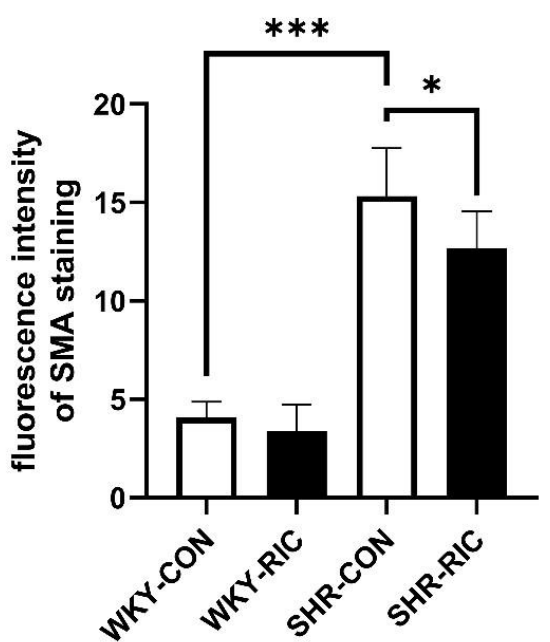

Figure 6. LRIC suppressed smooth muscle cell hypertrophy of small resistance artery. (A) Immunostaining of smooth muscle actin of the TA and MEA. SMC area (B) and fluorescence intensity (C) in TA of each group. SMC area (D) and fluorescence intensity (E) in MEA of each group. Data are expressed as mean $\pm \mathrm{SD}, * \mathrm{P}<0.05$, $* * \mathrm{P}<0.01, * * * \mathrm{P}<0.001$, WKY-CON vs. SHR-CON, SHR-CON vs. SHR-RIC. $\mathrm{N}=5$ each group. 
A
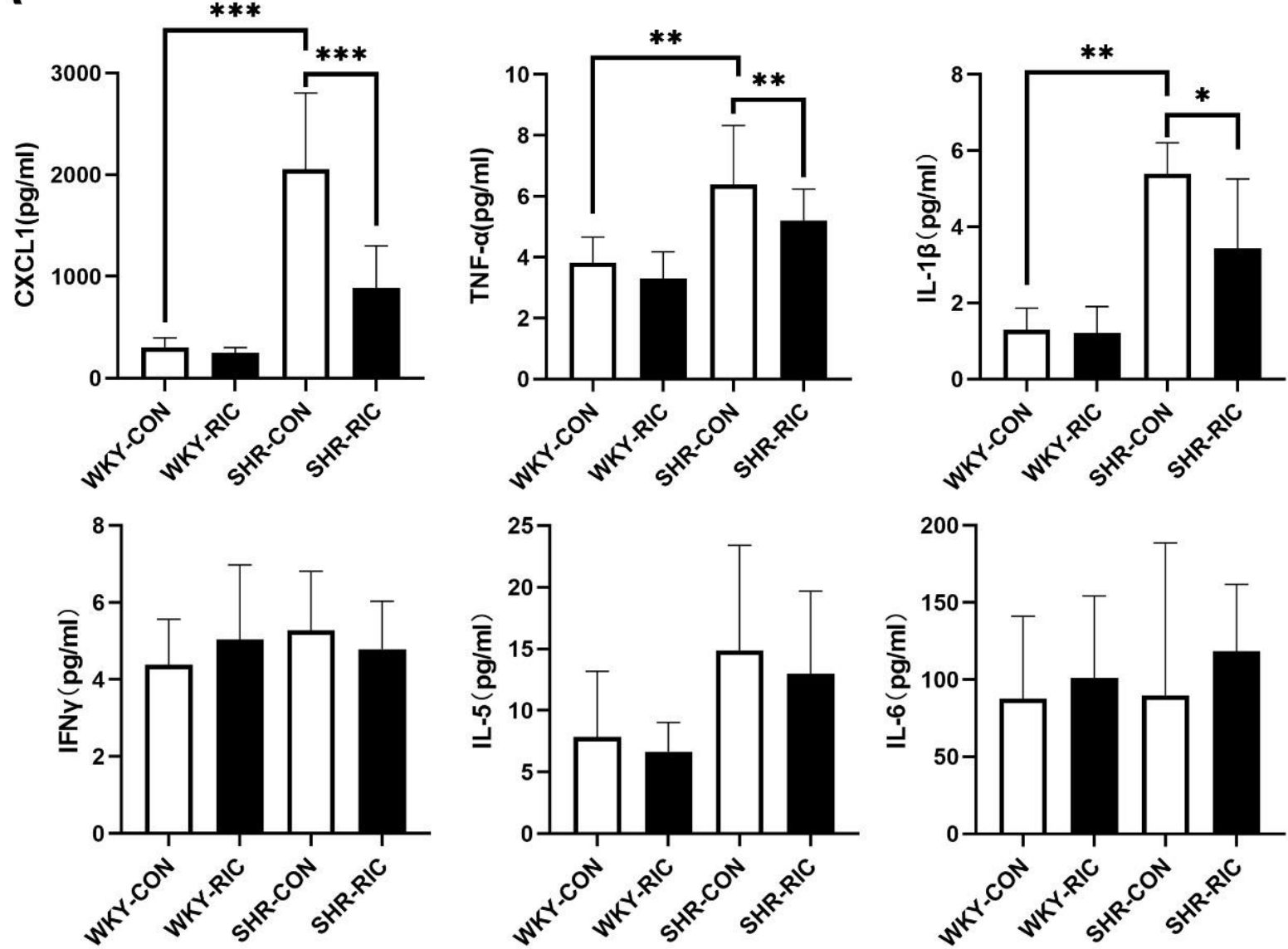

B
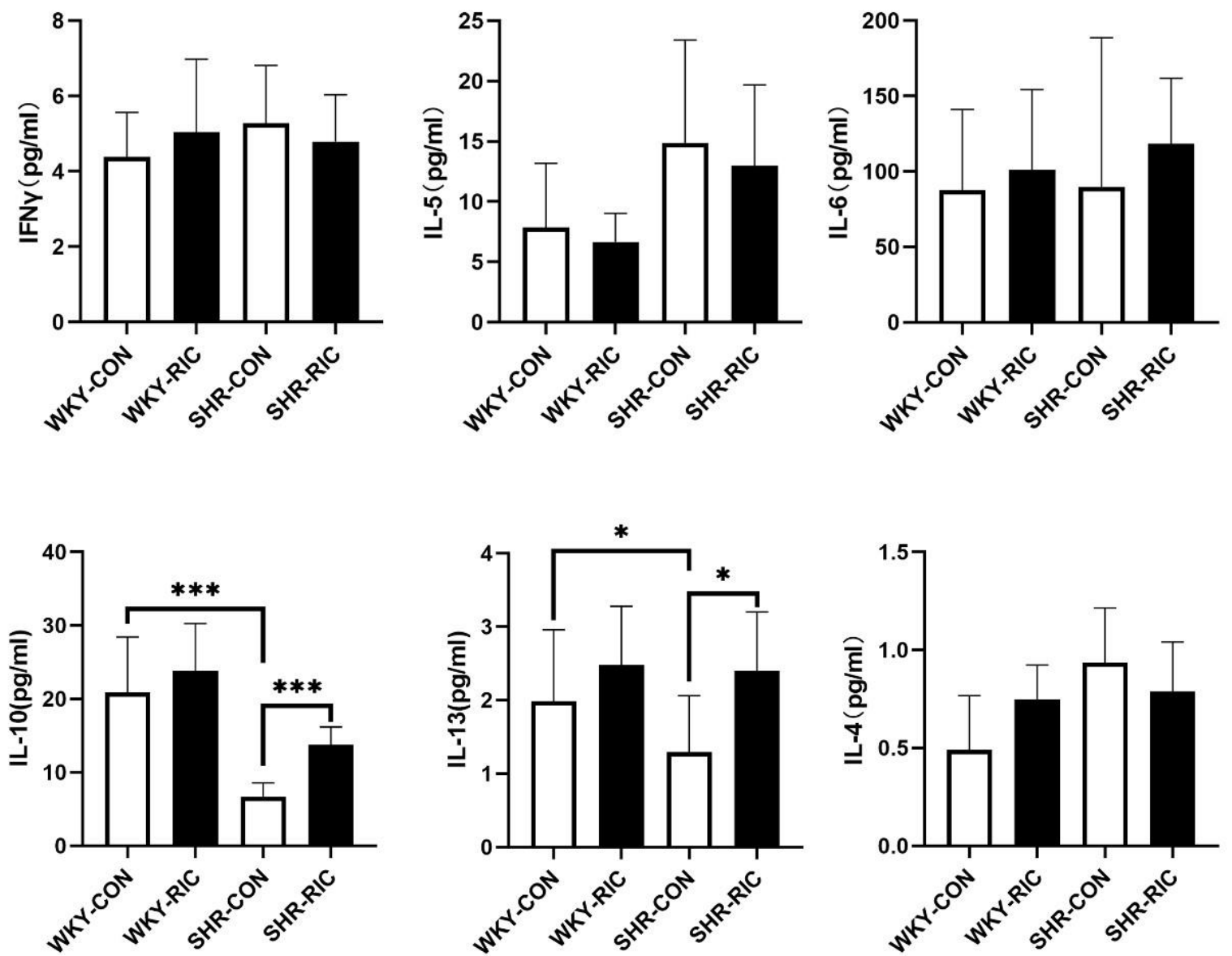

Figure 7. LRIC treatment regulated circulating pro-inflammatory and anti-inflammatory factors. (A) pro-inflammatory cytokines CXCL1, TNF $\alpha$, IL-1 $\beta$, IFN $\gamma$, IL-5, and IL-6 levels in plasma. (B) anti-inflammatory cytokines IL-10, IL-13, and IL-4 levels in plasma. Data are expressed as mean $\pm \mathrm{SD}, * \mathrm{P}<0.05, * * \mathrm{P}<0.01, * * * \mathrm{P}<0.001$, WKY-CON vs. SHR-CON, SHR-CON vs. SHR-RIC. $\mathrm{N}=5$ each group. 


\section{A}

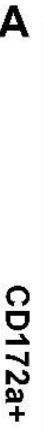
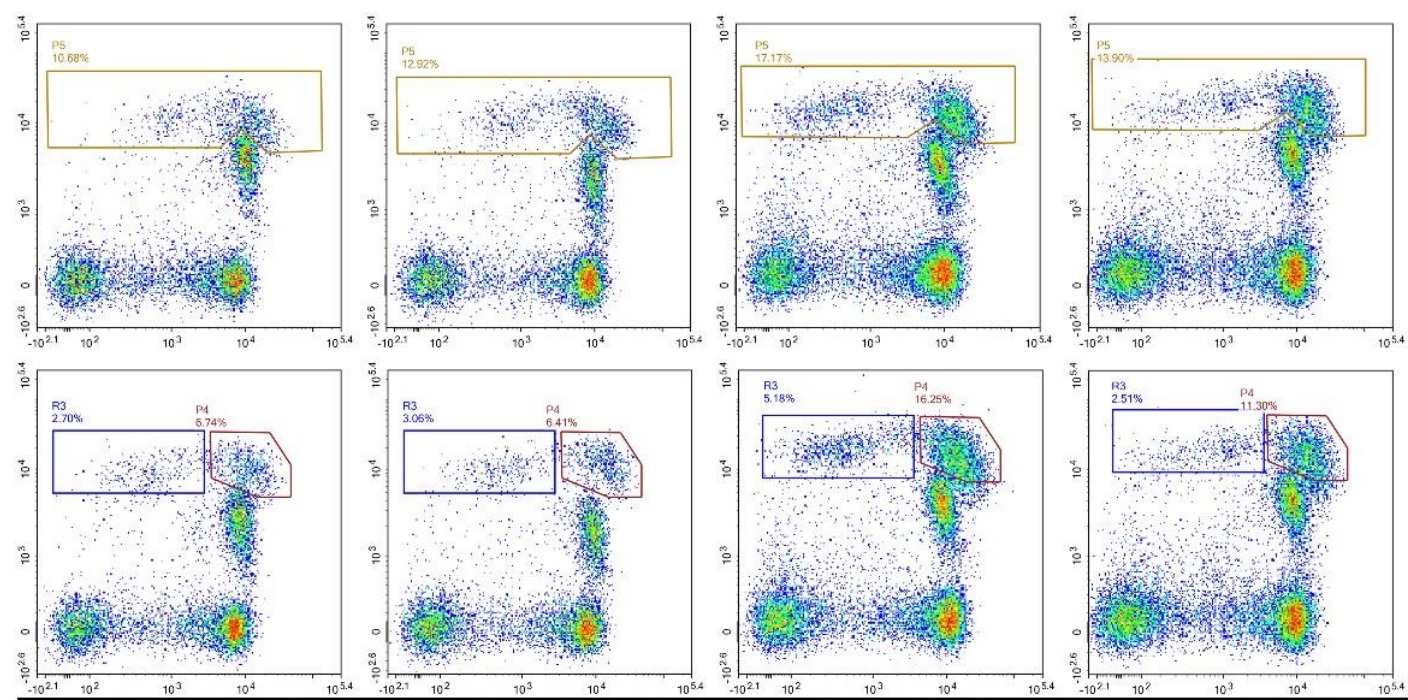

CD43+

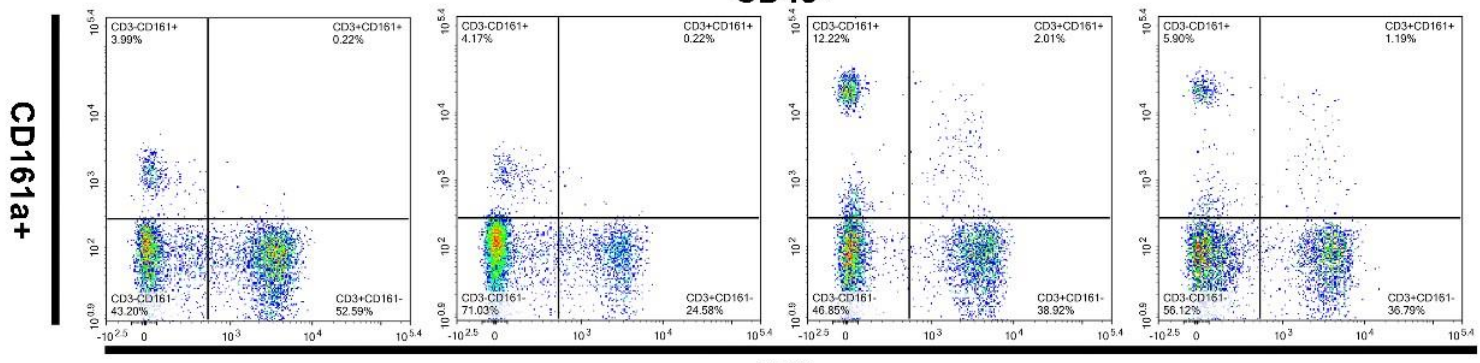

CD3+
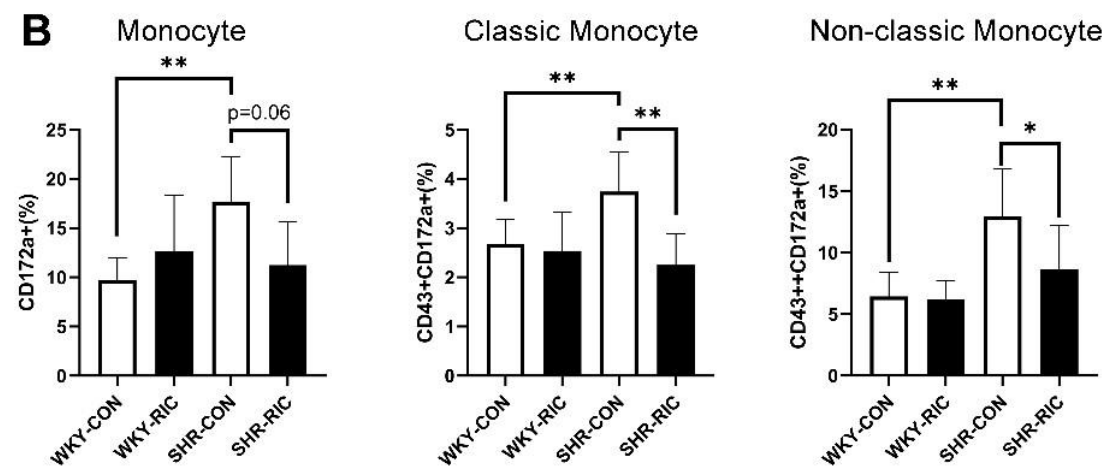

Natural Killer T Cell

Figure 8. LRIC treatment regulated inflammatory immunocytes in circulation. (A) Flow cytometry analysis of CD172a+, CD43+172a+, CD43++ 172a+, and CD3+CD161a+ cells in the circulation. (B) level of monocyte, classic monocyte, nonclassic monocyte, and natural killer T cells in circulation of each group. Data are expressed as mean $\pm \mathrm{SD}, * \mathrm{P}<0.05, * * \mathrm{P}<0.01$, $* * * \mathrm{P}<0.001, \mathrm{WKY}-\mathrm{CON}$ vs. SHR-CON, SHR-CON vs. SHR-RIC. $\mathrm{N}=5$ each group.

\section{DISCUSSION}

In this study, the results indicated that SHR develops hypertension coupled with vascular remodeling as early as 4-week old, as reported previously [17]. Long-term LRIC could decrease blood pressure to a certain extent; however, it could not reverse to normal levels. Histological staining results demonstrated that LRIC treatment ameliorated hypertrophic vascular remodeling of the conducting artery, resistance artery, and cerebral resistance artery, which contribute to considerable resistance to cerebral circulation than any other organ. 
LRIC could suppress hypertrophic vascular remodeling in two ways. One is to suppress the production of extracellular matrix; the other is to suppress SMA hypertrophy and hyperplasia. LRIC reduced inflammatory factors and increased anti-inflammatory factors in circulation. Consistent with the inflammatory analysis, LRIC reduced monocyte count in the blood, a source of inflammatory factors. In addition, LRIC decreased blood pressure and PWV, indicators of arterial remodeling [27]. Altogether, this study suggested that LRIC could lower blood pressure by regulating the circulating inflammatory cells and their markers to ameliorate hypertension-induced vascular remodeling. It is noteworthy that LRIC could decrease blood pressure and reduce arterial stiffness, thus offering a promising approach to the prevention of hypertension and vascular remodeling.
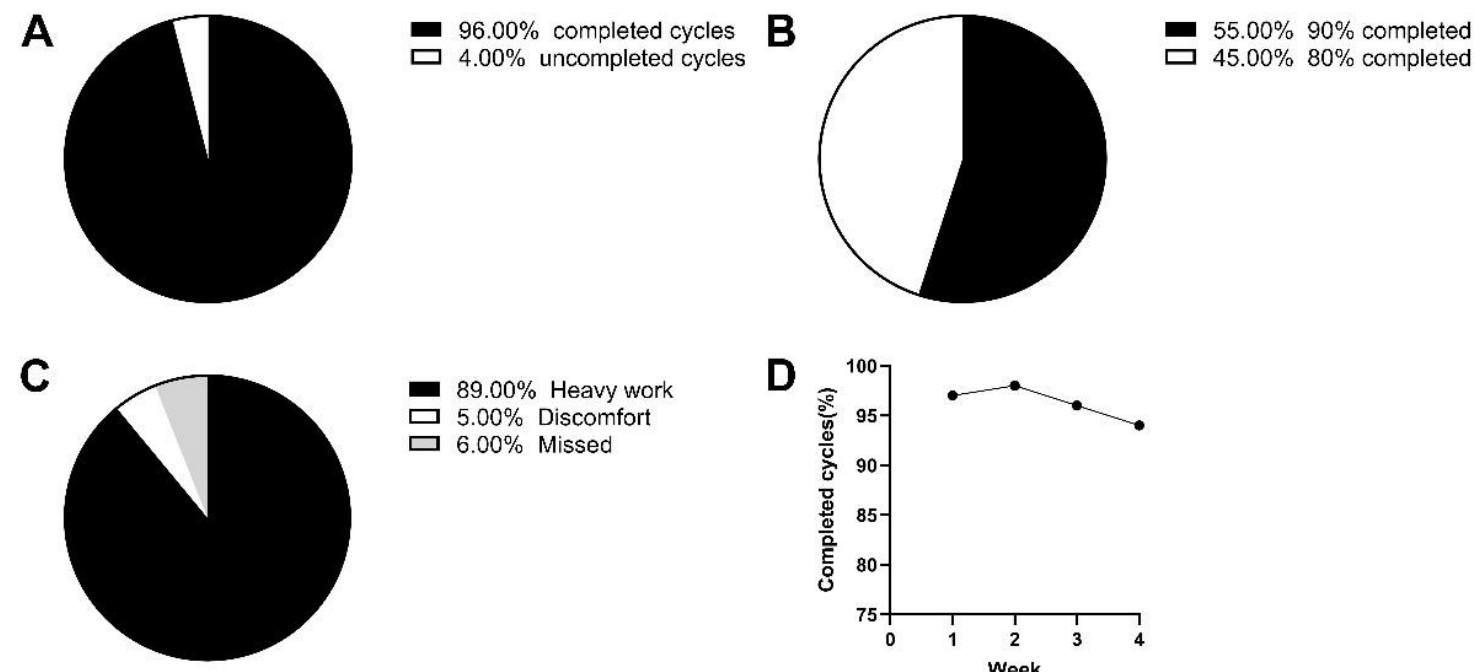

$89.00 \%$ Heavy work $5.00 \%$ Discomfort $6.00 \%$ Missed

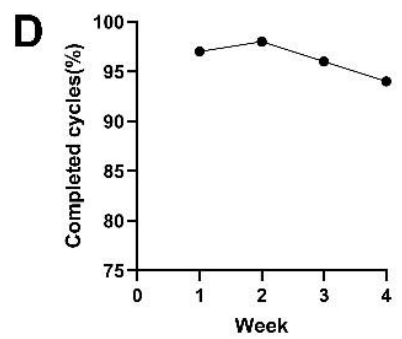

Figure 9. Hypertensive patient tolerance of LRIC. (A) The percentage of completed and uncompleted cycles. (B) The percentage of $90 \%$ and $80 \%$ completed patients. (C) The reasons for uncompleted cycles. (D) The percentage of completed cycles in each week.

Vascular remodeling underlies the pathological basis for all types of vascular diseases, and pathological remodeling helps establish stable hypertension [28]. Hypertensive vascular remodeling includes eutrophic remodeling and hypertrophic remodeling. Hypertrophic remodeling plays a central role in the development of hypertension and hypertensive end-organ injury [12]. For the conduit artery, hypertrophic remodeling is described as an outward increase of cross-sectional area and stiffness, similar to aging [29]. In the present study, we showed that LRIC could ameliorate the large artery remodeling primarily by suppressing deposition of the extracellular matrix. Consistent with the results, we found that long term LRIC could improve vascular stiffness in patients with pre- and early-stage hypertension. These results confirmed further that LRIC could protect the conducting artery from the pathological remodeling induced by hypertension or aging. The hypertrophic changes in the resistance artery refer mainly to an inward increase of cross-sectional area and decreased lumen, important components in end-organ damage, and most prevalent in early-stage hypertension [29, 30]. LRIC was shown to ameliorate the small resistance artery remodeling by suppressing the production of collagen and hypertrophy of smooth muscle cell. In addition, LRIC had a significant effect on the small resistance artery and cerebral muscular artery than that on the conduction artery. It is suggested that LRIC might be useful in the prevention and amelioration of diseases resulting from small artery remodeling like the cerebral small vessel disease [21].

Accumulating evidence demonstrates that increased circulating pro-inflammatory factors initiate vascular remodeling pathogenesis at an early-stage, typically accompanied by a decrease in anti-inflammatory factors [31]. Inflammation dysregulation could elevate blood pressure and trigger vascular remodeling. Suppressing pro-inflammatory factors or their receptors could decrease blood pressure and prevent vascular remodeling [32]. Hypertension is one such stimulus that initiates vascular remodeling mediated by inflammatory cell migration and infiltration [12]. 
A

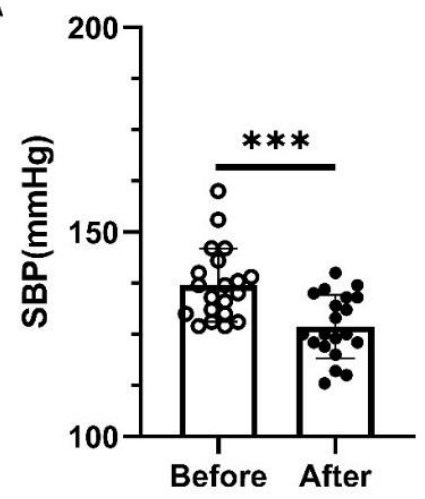

C

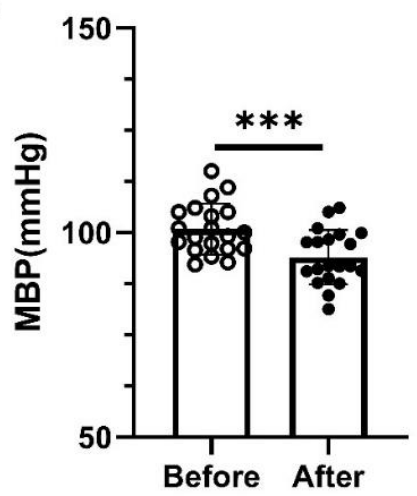

B

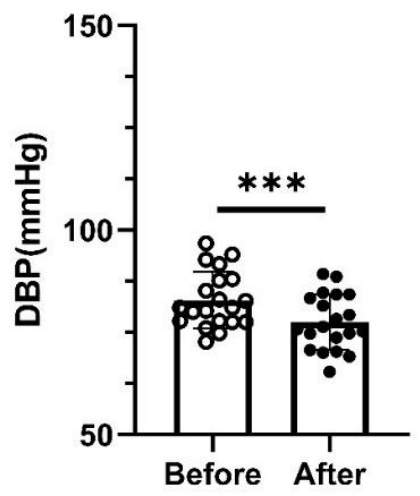

D

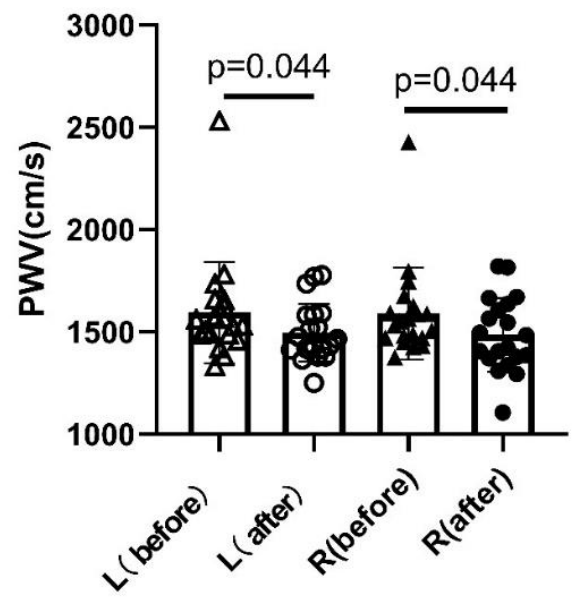

Figure 10. Long term LRIC decreased blood pressure and ameliorated conducting artery PWV. (A) Systolic blood pressure before and after LRIC treatment. (B) Diastolic blood pressure before and after LRIC treatment. (C) mean blood pressure before and after LRIC treatment. (D) PWV of left and right sides before and after LRIC treatment. $* \mathrm{P}<0.05, * * \mathrm{P}<0.01, * * * \mathrm{P}<0.001$, before vs. after.

CXCL1 is a chemokine that promotes migration and infiltration of neutrophils and monocytes to vascular wall[31]. The blockage of CXCR2, the receptor of CXCL1, could suppress hypertension and end-organ damage in SHR and other hypertensive animal models $[32,33]$. Our results showed that LRIC could significantly decrease plasma CXCL1 levels, suggesting that LRIC ameliorated vascular remodeling by decreasing CXCL1 production. TNF $\alpha$ has been reported to lead to the development of hypertension in various hypertensive models[34]. Anti-TNF $\alpha$ therapy reduced vascular inflammation and reduced arterial stiffness in patients with autoimmune diseases[35]. IL- $1 \beta$ levels were higher in hypertension and mediated vascular inflammation [36]. Consistent with previous results, our results demonstrated an increase in TNF $\alpha$ and IL-1 $\beta$ levels in SHR and LRIC reduced plasma TNF $\alpha$ and IL-1 $\beta$ levels, indicating that
LRIC exerts its effect by suppressing pro-inflammatory marker expression.

IL-10 is an anti-inflammatory cytokine exhibiting a protective effect on vascular dysfunction in hypertension [37]. A significant increase in IL-10 after kidney ischemic preconditioning was reported earlier[38]. IL-13 and IL-4 induced polarization of macrophage cells to an M2 phenotype, which expresses anti-inflammatory markers [39]. Previous research has found an imbalance in M1 and M2 population in SHR with an increased M2 macrophage population resulting in the normalization of blood pressure [40]. In this study, we found that LRIC increased circulating IL-10 and IL-13, which might participate in improving vascular function. All the findings in this study suggested that LRIC ameliorated vascular remodeling by suppressing the production of multiple pro-inflammatory factors while inducing the production of antiinflammatory factors in the circulation. Elevated 
inflammatory status exists in various chronic diseases, including aging [41], and LRIC opens a promising avenue for the prevention of aging and chronic diseases.

Circulating monocyte forming macrophages entering a specific organ or tissue plays a critical role in vascular inflammation and remodeling [42]. It is found that decreased number of lysozyme M-positive monocytes in circulation ablated the hypertensive stimuli induced vascular infiltration [11]. In addition, monocytes/ macrophages secrete pro-inflammatory factors and express receptors to interact with chemokines, such as CXCL1, TFN $\alpha$, and IL-1 $\beta$ [43]. Consistent with the inflammatory factor analysis, the monocyte counts in the circulation decreased after LRIC treatment. We also observed decreased classic monocyte counts compared to non-classic monocytes. Classic monocytes express proinflammatory clusters and produce pro-inflammatory factors, TNF $\alpha$ and IL-1 $\beta[44]$. From our results, it is suggested that LRIC could suppress the monocyte counts, especially the pro-inflammatory subset involved in hypertension and vascular remodeling. It is noteworthy that LRIC therapy could decrease the NKT cell level significantly. The relationship between NKT cells and hypertension has not been elucidated yet [45]. A previous study reported the association of NKT cells with cardiovascular events, while others demonstrated attenuation of vascular remodeling by a specialized NKT cell subset [46]. We showed markedly higher NKT cell counts in SHR compared to WKY, which decreased dramatically after LRIC treatment, thus shedding light on the novel mechanism of hypertension development.

Successful blood pressure management is critical to prevent cardiovascular and cerebrovascular disease. Some studies showed that sustained elevated blood pressure exceeding $125 \mathrm{mmHg}$ might represent a threshold for vascular remodeling, potential hypertension, and increased risk of future cardiovascular disease and stroke [47]. All the evidence highlights the importance of early prevention of blood pressure elevation. Our study results showed that LRIC could reduce the onset of blood pressure during pre- and early-stages in humans, suggesting that LRIC offers other means of hypertension and vascular dysfunction prevention for clinical translation.

However, some limitations exist in the human study. First, this is a single-arm study, and the date was not compared with the control group. It cannot exclude placebo effects, which may affect blood pressure. Instead, the data before and after ischemic conditioning were selfcompared. In addition, the method used to evaluate blood pressure is not precise, and the effect on blood pressure should be explored in a longer time frame. Thus, the results from the human study are implied and are being investigated further in a randomized, double-blind placebo control study (Registration No. NCT03566654, https://register.clinicaltrials.gov).

In conclusion, long-term LRIC treatment could decrease blood pressure and attenuate vascular remodeling in SHR. LRIC exerted its protective effect by restoring the imbalance in pro- and anti-inflammatory markers. These findings describe the potential use of LRIC for the prevention of hypertension.

\section{Acknowledgments}

This work was supported by the Natural Science Foundation of China (No. 81971114, No. 81573867, No. 81801313), Science and technology development project of Beijing Municipal Health Commission, and the National Key R\&D Program of China (2017YFC 1308402).

\section{Conflicts of Interest}

The authors declare no conflict of interest.

\section{References}

[1] Whelton PK, Carey RM, Aronow WS, Casey DE, Jr., Collins KJ, Dennison Himmelfarb C, et al. (2018). 2017 ACC/AHA/AAPA/ABC/ACPM/AGS/APhA/ASH/ASP C/NMA/PCNA Guideline for the Prevention, Detection, Evaluation, and Management of High Blood Pressure in Adults: Executive Summary: A Report of the American College of Cardiology/American Heart Association Task Force on Clinical Practice Guidelines. J Am Coll Cardiol, 71:2199-2269.

[2] Benjamin EJ, Blaha MJ, Chiuve SE, Cushman M, Das SR, Deo R, et al. (2017). Heart Disease and Stroke Statistics-2017 Update: A Report From the American Heart Association. Circulation, 135:e146-e603.

[3] Iadecola C, Davisson RL (2008). Hypertension and cerebrovascular dysfunction. Cell Metab, 7:476-484.

[4] Laurent S, Boutouyrie P (2015). The structural factor of hypertension: large and small artery alterations. Circ Res, 116:1007-1021.

[5] Intengan HD, Schiffrin EL (2001). Vascular remodeling in hypertension: roles of apoptosis, inflammation, and fibrosis. Hypertension, 38:581-587.

[6] Schiffrin EL, Touyz RM (2004). From bedside to bench to bedside: role of renin-angiotensin-aldosterone system in remodeling of resistance arteries in hypertension. Am J Physiol Heart Circ Physiol, 287:H435-446.

[7] Niiranen TJ, Larson MG, McCabe EL, Xanthakis V, Vasan RS, Cheng S (2017). Prognosis of Prehypertension Without Progression to Hypertension. Circulation, 136:1262-1264.

[8] Xu X, Wang B, Ren C, Hu J, Greenberg DA, Chen T, et al. (2017). Age-related Impairment of Vascular Structure and Functions. Aging Dis, 8:590-610.

[9] Renna NF, de Las Heras N, Miatello RM (2013). 
Pathophysiology of vascular remodeling in hypertension. Int J Hypertens, 2013:808353.

[10] Guzik TJ, Touyz RM (2017). Oxidative Stress, Inflammation, and Vascular Aging in Hypertension. Hypertension, 70:660-667.

[11] Wenzel P, Knorr M, Kossmann S, Stratmann J, Hausding M, Schuhmacher S, et al. (2011). Lysozyme M-positive monocytes mediate angiotensin II-induced arterial hypertension and vascular dysfunction. Circulation, 124:1370-1381.

[12] McMaster WG, Kirabo A, Madhur MS, Harrison DG (2015). Inflammation, immunity, and hypertensive endorgan damage. Circ Res, 116:1022-1033.

[13] Heusch G, Botker HE, Przyklenk K, Redington A, Yellon D (2015). Remote ischemic conditioning. J Am Coll Cardiol, 65:177-195.

[14] Li S, Hafeez A, Noorulla F, Geng X, Shao G, Ren C, et al. (2017). Preconditioning in neuroprotection: From hypoxia to ischemia. Prog Neurobiol, 157:79-91.

[15] Zhao W, Li S, Ren C, Meng R, Ji X (2018). Chronic Remote Ischemic Conditioning May Mimic Regular Exercise:Perspective from Clinical Studies. Aging Dis, 9:165-171.

[16] Madias JE (2015). Sustained blood pressure lowering effect of twice daily remote ischemic conditioning sessions in a normotensive/prehypertensive subject. Int J Cardiol, 182:392-394.

[17] Limas C, Westrum B, Limas CJ (1980). The evolution of vascular changes in the spontaneously hypertensive rat. Am J Pathol, 98:357-384.

[18] Zhang X, Jizhang Y, Xu X, Kwiecien TD, Li N, Zhang $\mathrm{Y}$, et al. (2014). Protective effects of remote ischemic conditioning against ischemia/reperfusion-induced retinal injury in rats. Vis Neurosci, 31:245-252.

[19] Taltavull N, Munoz-Cortes M, Lluis L, Jove M, Fortuno A, Molinar-Toribio E, et al. (2014). Eicosapentaenoic acid/docosahexaenoic acid 1:1 ratio improves histological alterations in obese rats with metabolic syndrome. Lipids Health Dis, 13:31.

[20] Ren C, Wu H, Li D, Yang Y, Gao Y, Jizhang Y, et al. (2018). Remote Ischemic Conditioning Protects Diabetic Retinopathy in Streptozotocin-induced Diabetic Rats via Anti-Inflammation and Antioxidation. Aging Dis, 9:1122-1133.

[21] Wang Y, Meng R, Song H, Liu G, Hua Y, Cui D, et al. (2017). Remote Ischemic Conditioning May Improve Outcomes of Patients With Cerebral Small-Vessel Disease. Stroke, 48:3064-3072.

[22] Pires PW, Dams Ramos CM, Matin N, Dorrance AM (2013). The effects of hypertension on the cerebral circulation. Am J Physiol Heart Circ Physiol, 304:H1598-1614.

[23] Ren C, Li N, Li S, Han R, Huang Q, Hu J, et al. (2018). Limb Ischemic Conditioning Improved Cognitive Deficits via eNOS-Dependent Augmentation of Angiogenesis after Chronic Cerebral Hypoperfusion in Rats. Aging Dis, 9:869-879.

[24] Briones AM, Arribas SM, Salaices M (2010). Role of extracellular matrix in vascular remodeling of hypertension. Curr Opin Nephrol Hypertens, 19:187-194.
[25] Olivetti G, Melissari M, Marchetti G, Anversa P (1982). Quantitative structural changes of the rat thoracic aorta in early spontaneous hypertension. Tissue composition, and hypertrophy and hyperplasia of smooth muscle cells. Circ Res, 51:19-26.

[26] Moser B, Wolf M, Walz A, Loetscher P (2004). Chemokines: multiple levels of leukocyte migration control. Trends Immunol, 25:75-84.

[27] Farrar DJ, Bond MG, Riley WA, Sawyer JK (1991). Anatomic correlates of aortic pulse wave velocity and carotid artery elasticity during atherosclerosis progression and regression in monkeys. Circulation, 83:1754-1763.

[28] Baeyens N, Bandyopadhyay C, Coon BG, Yun S, Schwartz MA (2016). Endothelial fluid shear stress sensing in vascular health and disease. J Clin Invest, 126:821-828.

[29] Schiffrin EL (2007). The vascular phenotypes in hypertension: Relation with the natural history of hypertension. J Am Soc Hypertens, 1:56-67.

[30] Schiffrin EL (2004). Vascular stiffening and arterial compliance. Implications for systolic blood pressure. Am J Hypertens, 17:39S-48S.

[31] Martynowicz H, Janus A, Nowacki D, Mazur G (2014). The role of chemokines in hypertension. Adv Clin Exp Med, 23:319-325.

[32] Wang L, Zhao XC, Cui W, Ma YQ, Ren HL, Zhou X, et al. (2016). Genetic and Pharmacologic Inhibition of the Chemokine Receptor CXCR2 Prevents Experimental Hypertension and Vascular Dysfunction. Circulation, 134:1353-1368.

[33] Zhang YL, Geng C, Yang J, Fang J, Yan X, Li PB, et al. (2019). Chronic inhibition of chemokine receptor CXCR2 attenuates cardiac remodeling and dysfunction in spontaneously hypertensive rats. Biochim Biophys Acta Mol Basis Dis, 1865:165551.

[34] Tran LT, MacLeod KM, McNeill JH (2009). Chronic etanercept treatment prevents the development of hypertension in fructose-fed rats. Mol Cell Biochem, 330:219-228.

[35] Maki-Petaja KM, Elkhawad M, Cheriyan J, Joshi FR, Ostor AJ, Hall FC, et al. (2012). Anti-tumor necrosis factor-alpha therapy reduces aortic inflammation and stiffness in patients with rheumatoid arthritis. Circulation, 126:2473-2480.

[36] Krishnan SM, Sobey CG, Latz E, Mansell A, Drummond GR (2014). IL-1beta and IL-18: inflammatory markers or mediators of hypertension? $\mathrm{Br} \mathrm{J}$ Pharmacol, 171:5589-5602.

[37] Lima VV, Zemse SM, Chiao CW, Bomfim GF, Tostes RC, Clinton Webb R, et al. (2016). Interleukin-10 limits increased blood pressure and vascular RhoA/Rho-kinase signaling in angiotensin II-infused mice. Life Sci, 145:137-143.

[38] Kinsey GR, Huang L, Vergis AL, Li L, Okusa MD (2010). Regulatory $\mathrm{T}$ cells contribute to the protective effect of ischemic preconditioning in the kidney. Kidney Int, 77:771-780.

[39] Zhang MZ, Wang X, Wang Y, Niu A, Wang S, Zou C, et al. (2017). IL-4/IL-13-mediated polarization of renal 
macrophages/dendritic cells to an M2a phenotype is essential for recovery from acute kidney injury. Kidney Int, 91:375-386.

[40] Harwani SC (2018). Macrophages under pressure: the role of macrophage polarization in hypertension. Transl Res, 191:45-63.

[41] Flynn MG, Markofski MM, Carrillo AE (2019). Elevated Inflammatory Status and Increased Risk of Chronic Disease in Chronological Aging: Inflamm-aging or Inflamm-inactivity? Aging Dis, 10:147-156.

[42] Boyette LB, Macedo C, Hadi K, Elinoff BD, Walters JT, Ramaswami B, et al. (2017). Phenotype, function, and differentiation potential of human monocyte subsets. PLoS One, 12:e0176460.

[43] Yang J, Zhang L, Yu C, Yang XF, Wang H (2014). Monocyte and macrophage differentiation: circulation inflammatory monocyte as biomarker for inflammatory diseases. Biomark Res, 2:1.

[44] Franca CN, Izar MCO, Hortencio MNS, do Amaral JB, Ferreira CES, Tuleta ID, et al. (2017). Monocyte subtypes and the CCR2 chemokine receptor in cardiovascular disease. Clin Sci (Lond), 131:1215-1224.

[45] Wenzel U, Turner JE, Krebs C, Kurts C, Harrison DG, Ehmke H (2016). Immune Mechanisms in Arterial Hypertension. J Am Soc Nephrol, 27:677-686.

[46] Wang HX, Li WJ, Hou CL, Lai S, Zhang YL, Tian C, et al. (2019). CD1d-dependent natural killer $\mathrm{T}$ cells attenuate angiotensin II-induced cardiac remodelling via IL-10 signalling in mice. Cardiovasc Res, 115:83-93.

[47] Niiranen TJ, Henglin M, Claggett B, Muggeo VMR, McCabe E, Jain M, et al. (2018). Trajectories of Blood Pressure Elevation Preceding Hypertension Onset: An Analysis of the Framingham Heart Study Original Cohort. JAMA Cardiol, 3:427-431. 